\title{
Fractal analyses: statistical and methodological innovations and best practices
}

\author{
John G. Holden ${ }^{1 *}$, Michael A. Riley ${ }^{2}$, Jianbo Gao ${ }^{3,4,5}$ and Kjerstin Torre ${ }^{6}$ \\ 1 Complexity Group, Department of Psychology, CAP center for Cognition, Action, and Perception, University of Cincinnati, Cincinnati, OH, USA \\ ${ }^{2}$ Department of Psychology, CAP Center for Cognition, Action, and Perception, University of Cincinnati, Cincinnati, OH, USA \\ ${ }^{3}$ PMB Intelligence LLC, West Lafayette, IN, USA \\ ${ }^{4}$ Department of Mechanical and Materials Engineering, Wright State University, Dayton, OH, USA \\ ${ }^{5}$ State Key Laboratory of Non-linear Mechanics, Institute of Mechanics, Chinese Academy of Sciences, Beijing, China \\ ${ }^{6}$ Movement to Health, University Montpellier, Montpellier, France \\ *Correspondence: holdenjn@ucmail.uc.edu
}

Edited by:

Bruce J. West, U.S. Army Research Laboratory, USA

Reviewed by:

Bruce J. West, U.S. Army Research Laboratory, USA

Fractal statistics now routinely appear in the scientific literature. Examples originate from many disciplines, including aquatic sciences, biology, computer science, ecology, economics, geology, mathematics, medicine, neuroscience, physics, physiology, and psychology. This eBook provides a broad range of resources to support the application of fractal methods and theory in physiology and related disciplines. It is comprised of a set of research topic articles that appeared in the Frontiers in Physiology specialty section: Fractal Physiology. Our eBook chapters are organized along a loose continuum defined by the characteristics of the empirical measurements a given statistical technique is intended to confront.

At one end of the continuum are techniques designed for application to stochastic systems. van Rooij et al. (2013) describe histograms, probability distributions, and scaling distributions in fractal terms. The next step on the continuum concerns self-affine random fractals and methods intended for outcome measures that entail scale-invariant $1 / f$ patterns or related patterns of temporal fluctuation. Stadnitski (2012) overviews several statistical procedures available for the analysis of fractal time-series measurements. Riley et al. (2012) discuss an adaptive fractal analysis that broadens the potential range of bio-signals that can be understood from a fractal perspective. Likewise, Marmelat et al.

\section{REFERENCES}

Eke, A., Herman, P., Sanganahalli, B. G., Hyder, F., Mukli, P., and Nagy, Z. (2012). Pitfalls in fractal time series analysis: fMRI BOLD as an exemplary case. Front. Physiol. 3:417. doi: 10.3389/fphys.2012.00417

Gao, J., Hu, J., Tung, W.-W., and Blasch, E. (2012). Multiscale analysis of biological data by scale-dependent Lyapunov exponent. Front. Physiol. 2:110. doi: 10.3389/fphys.2011.00110

Hasselman, F. (2013). When the blind curve is finite: dimension estimation and model inference based on empirical waveforms. Front. Physiol. 4:75. doi: 10.3389/fphys.2013.00075

Ihlen, E. A. (2012). Introduction to multifractal detrended fluctuation analysis in Matlab. Front. Physiol.
3:141. doi: 10.3389/fphys.2012. 00141

Marmelat, V., Torre, K., and Delignières, D. (2012). Relative roughness: an index for testing the suitability of the monofractal model. Front. Physiol. 3:208. doi: 10.3389/fphys.2012.00208

Richardson, M. J., Garcia, R. L., Frank, T. D., Gergor, M., and Marsh, K. L. (2012). Measuring group synchrony: a cluster-phase method for analyzing multivariate movement time-series. Front. Physiol. 3:405. doi: 10.3389/fphys.2012.00405

Riley, M. A., Bonnette, S., Kuznetsov, N., Wallot, S., and Gao, J. (2012). A tutorial introduction to adaptive fractal analysis. Front. Physiol. 3:371. doi: 10.3389/fphys.2012.00371

(2012) illustrate a relative roughness scale, helpful in determining the applicability of a monofractal description to a given signal. Wijnants et al. (2013) examines how of signal sampling rate artifacts influence spectrally derived scaling exponents. Hasselman (2013) discusses relationships among a set of common fractal time-series analyses, and advocates reliance on theory-driven predictions as a route to understanding the systems that yield empirical patterns. Eke et al. (2012) bridge the monofractal and multifractal frameworks with a special emphasis on the appropriate and accurate characterization of measured signals. Ihlen (2012) supplies a detailed tutorial on multifractal detrended fluctuation analysis.

The deterministic end of the statistical continuum emphasizes techniques used to investigate systems that express differentiable trajectories. Webber (2012) illustrates recurrence analysis on time-series derived from several multi-dimensional dynamic systems. Gao et al. (2012) introduces a very general analysis that is suitable for use on both stochastic and continuous measurements. Finally, Richardson et al. (2012) describe techniques that assess relative dynamic synchrony among multiple coupled oscillatory time-series. Taken together, the chapters offer a gamut of analytic strategies alongside contemporary expertise on how to best conduct and interpret the outcomes of fractal analyses.

Stadnitski, T. (2012). Measuring fractality. Front. Physiol. 3:127. doi: 10.3389/fphys.2012.00127

van Rooij, M. M. J. W., Nash, B. A., Rajaraman, S., and Holden, J. G. (2013). A fractal approach to dynamic inference and distribution analysis. Front. Physiol. 4:1. doi: 10.3389/fphys.2013.00001

Webber, C. L. Jr. (2012). Recurrence quantification of fractal structures. Front. Physiol. 3:382. doi: 10.3389/fphys.2012.00382

Wijnants, M. L., Cox, R. F. A. Hasselman, F., Bosman, A. M. T., and Van Orden, G. (2013). Does sample rate introduce an artifact in spectral analysis of continuous processes? Front. Physiol. 3:495. doi: 10.3389/fphys. 2012.00495
Received: 17 April 2013; accepted: 20 April 2013; published online: 08 May 2013.

Citation: Holden JG, Riley MA, Gao J and Torre K (2013) Fractal analyses: statistical and methodological innovations and best practices. Front. Physiol. 4:97. doi: 10.3389/fphys.2013.00097

This article was submitted to Frontiers in Fractal Physiology, a specialty of Frontiers in Physiology.

Copyright (c) 2013 Holden, Riley, Gao and Torre. This is an open-access article distributed under the terms of the Creative Commons Attribution License, which permits use, distribution and reproduction in other forums, provided the original authors and source are credited and subject to any copyright notices concerning any third-party graphics etc. 